\title{
TWO NOTES ON RANKIN'S BOOK ON THE MODULAR GROUP
}

\author{
Dedicated to the memory of Hanna Neumann
}

ROGER LYNDON

(Received 27 June 1972)

Communicated by M. F. Newman

We supply proofs that are simple, and possibly partly new, for two theorems that appear in Rankin's book [6].

1.

The first concerns subgroups of the inhomogeneous modular group. Let $\Gamma=S L(2, Z)$, the group of all 2 by 2 matrices with integer coefficients and with determinant 1. For each positive integer $n$, let $\Gamma(n)$ consist of those $T$ in $\Gamma$ such that $T \equiv I$ modulo $n$. Let $\Delta(n)$ be the least normal subgroup of $\Gamma$ that contains the element $\left(\begin{array}{ll}1 & n \\ 0 & 1\end{array}\right)$.

THEOREM 1. (a) If $1 \leqq n \leqq 5$, then $\Gamma(n)=\Delta(n)$.

(b) For all $n$, the index of $\Gamma(n)$ in $\Gamma$ is $\eta(n)=n^{3} \prod_{p / n}\left(1-1 / p^{2}\right)$.

(c) For $n \geqq y$, the index of $\Delta(n)$ in $\Gamma$ is infinite.

We first prove (b). Note that both $\Gamma(n)$ and $\Delta(n)$ are normal in $\Gamma$; thus we may write $\Gamma_{n}=\Gamma / \Gamma(n)$ and $\Delta_{n}=\Gamma / \Delta(n)$. Observe that both $\left|\Gamma_{n}\right|$ and $\eta(n)$ are multiplicative functions of $n$; therefore it suffices to treat the case that $n$ is a power of a prime. The routine solution of a system of congruences shows that, under the natural map, $\Gamma_{n} \cong S L\left(2, Z_{n}\right)$. A standard argument gives the order of the latter group.

Clearly $\Delta(n) \subseteq \Gamma(n)$, whence $\left|\Gamma_{n}\right| \leqq\left|\Delta_{n}\right|$. Therefore, to establish (a) and (c) it suffices to show that, for $n \leqq 5,\left|\Delta_{n}\right| \leqq \eta(n)$ and that for $n \geqq 6,\left|\Delta_{n}\right|=\infty$. In the sequel we put aside the trivial case that $n=1$.

Both $\Gamma$ and $\Delta_{n}$ have center $Z=\{I,-I\}$. It is well known that $P=\Gamma / Z$ has the presentation

$$
P\left\langle A, B: A^{2}=1, B^{3}=1\right\rangle,
$$

where $A$ and $B$ are given by the matrices $\left(\begin{array}{rr}0 & -1 \\ 1 & 0\end{array}\right)$ and $\left(\begin{array}{lr}0 & -1 \\ 1 & 1\end{array}\right)$. Since $(A B)^{n}$ is 
given by $\left(\begin{array}{ll}1 & n \\ 0 & 1\end{array}\right)$, the group $G_{n}=\Delta_{n} / Z$ obtained from $P$ by imposing the further relation $(A B)^{n}=1$ has the presentation

$$
G_{n}=\left\langle A, B ; A^{2}=1, B^{3}=1,(A B)^{n}=1\right\rangle .
$$

These groups are well known, especially from the work of Coxeter (see [1]). It. remains to show that $G_{2}$ has order $\eta(2)$, that $2\left|G_{n}\right|=\eta(n)$ for $n=3,4,5$, and that $G_{n}$ is infinite for $n \geqq 6$.

For each $n \geqq 2$ we shall construct a graph $T_{n}$ on the sphere (or better, for $n \geqq 6$, in the plane) such that each region is a triangle and that there are exactly $n$ edges at each vertex. For $n=2,3,4,5$ we take $T_{n}$ to be a triangle, the 1-skeleton of a tetrahedron, of an octahedron, of an icosahedron. This reflects the fact that the corresponding $\Delta_{n}$ are the symmetry groups of these figures.

Let $n \geqq 6$. We begin with a triangle $A B C$ and enclose it it a circle $K$ not touching it. Join $A$ and $B$ to a point $C^{\prime}$ on $K$, join $B$ and $C$ to a point $A^{\prime}$ on $K$, and join $C$ and $A$ to a point $B^{\prime}$ on $K$. Now join each of $A, B$, and $C$ to additional points on $K$ until there are $n$ edges at each of these three vertices. Clearly this can be done so that each bounded region in the resulting figure is a (topological) triangle. We now enclose the figure obtained so far in a larger circle $K^{\prime}$ not touching it, and repeat the process. It is easy to see that this process can be iterated indefinitely and will yield an infinite graph $T_{n}$ with the required properties.

We now modify $T_{n}$ to obtain a new graph $S_{n}$. We draw small circles about the vertices of $T_{n}$ (small enough that no two meet) and delete those parts of $T_{n}$ interior to these circles. In $S_{n}$ each vertex lies on exactly three edges: two that are arcs of the small circles and one that is 'straight', that is, the remnant of an edge from $T_{n}$.

Let $\Omega$ be the set of vertices of $S_{n}$. Define two permutations of $\Omega$ as follows. If $P$ is any vertex, then $\alpha(P)$ is the other vertex on the straight edge at $P$, while $\gamma(P)$ is the other vertex on the circular arc proceeding counterclockwise out of $P$. It is immediate that $\alpha^{2}=1$ and $\gamma^{n}=1$. Moreover, inspection shows that, if $\beta=\alpha \gamma$, then $\beta^{3}=1$. If $\pi$ is the group of permutations of $\Omega$ generated by $\alpha$ and $\gamma$, it is immediate that setting $\phi(A)=\alpha$ and $\phi(B)=\beta$ define a homomorphism $\phi$ from $G_{n}$ onto $\pi$. (In fact $\phi$ is an isomorphism, and $S_{n}$ is a Cayley diagram for $G_{n}$; alternatively, that $\phi$ is an isomorphism follows by a method known to Poincaré (see Macbeath [3]).

Since $\pi$ acts regularly on $\Omega$, we have $|\pi|=|\Omega|$, whence $\left|G_{n}\right| \geqq|\Omega|$. This gives the desired inequality for $\left|\Delta_{n}\right|$ in both the finite and infinite cases.

2.

The second theorem is one of Nielsen [4]. We state it in a mildly modified form.

THEOREM 2. Let $G_{1}, \cdots, G_{n}$ be arbitrary groups, and let $N$ be the kernel 
of the natural map from the free product $G=G_{1} * \cdots * G_{n}$ onto the direct product $G=G_{1} \times \cdots \times G_{n}$. Then $N$ is free group with a basis $X$ consisting of all non trivial elements of the form

$$
x=\left(a_{1} \cdots a_{i-1} a_{i+1} \cdots a_{n} a_{i}\right)\left(a_{1} \cdots a_{n}\right)^{-1}
$$

where $a_{1} \in G_{1}, \cdots, a_{n} \in G_{n}$.

We begin the proof by showing that $X$ generates $N$. Let $H$ be the subgroup generated by $X$. Clearly $H \subseteq N$. It will suffice to show that $G=H G_{1} \cdots G_{n}$. For this it suffices to show that, for all $i=1.2, \cdots, n$ one has $H G_{1} \cdots G_{n} G_{i}=H G_{1} \cdots G_{n}$. Now $H x=H$ for all $x \in X$ implies that $H G_{1} \cdots G_{i-1} G_{i+1} \cdots G_{n} G_{i}=H G_{1} \cdots G_{n}$. Using this relation we find that $H G_{1} \cdots G_{n} G_{i}=H G_{1} \cdots G_{i-1} G_{i+1} \cdots G_{n} G_{i} G_{i}$ $H G_{1} \cdots G_{i-1} G_{i+1} \cdots G_{n} G_{i}=H G_{1} \cdots G_{n}$.

It remains to show that $X$ is a basis for $N$. Note that an element $x$ as above is not trivial just in case $a_{i} \neq 1$ and that $a_{j} \neq 1$ for some $j>i$. We write $x=U V^{-1}$ where $U=a_{1} \cdots a_{i-1} a_{i+1} \cdots a_{n} a_{i}$ and $V=a_{1} \cdots a_{n}$. Then $U$ and $V$ have the same length $|U|=|V|=m \leqq n$ and $|x|=2 m$. (Here $m$ is the number of non-trivial factors $a_{i}$.) Let $x^{\prime}=U^{\prime} V^{\prime-1}$ denote analogously another element of $X$.

We make several observations.

(1) If $x$ and $x^{\prime}$ have the initial segment $U$ in common, then $x=x^{\prime}$. This follows from the fact that $a_{i}$, as the first (non trivial) syllable of $x$ with decreasing subscript, must match the first such syllable of $x^{\prime}$. This implies that $U=U^{\prime}$ whence also $V=V^{\prime}$.

(2) If $x^{-1}$ and $x^{-1}$ have an initial segment longer than $V$ in common, then $x=x^{\prime}$. Suppose they had such an initial segment in common, and hence the initial segment $V a_{i}^{-1}$. Since $a_{i}^{-1}$ is the first syllable of $x^{-1}$ with decreasing subscript, it must match the first such syllable of $x^{-1}$, whence $V a_{i}^{-1}=V^{\prime} a_{i^{\prime}}^{-1}$. From $V=V^{\prime}$ it follows that $a_{1}=a_{1}^{\prime}, \cdots, a_{n}=a_{n}^{\prime}$, and we have also that $a_{i}^{-1}=a_{i^{\prime}}^{-1}$, whence $i=i^{\prime}$. Thus $U=U^{\prime}$ and $x=x^{\prime}$.

(3) If $x^{-1}$ and $x^{\prime}$ have the initial segment $V$ in common, then they have no longer initial segment in common, and the segment $V$ is less than half of $x^{\prime}$. This follows from the facts that $V$, with increasing subscripts, must be a proper initial segment of $U^{\prime}$, and that $V a_{i}^{-1}$, containing two syllables $a_{i}$ and $a_{i}^{-1}$ from $G_{i}$, cannot be a segment of $U^{\prime}$.

(4) In a product $x x^{\prime e} \neq 1$, with $e= \pm 1$, at most the right half $V^{-1}$ of $x$ cancels. If $e=+1$ this follows from (3), and if $e=-1$ from (2).

(5) In a product $x^{\prime e} x \neq 1$, with $e= \pm 1$, not all of the left half $U$ of $x$ cancels. If $e=+1$ this follows from (3), and if $e=-1$ from (1).

Now a classical argument of Nielsen [5] shows that in a product $w=$ $x_{1}^{e_{1}} \cdots x_{k}^{e_{k}}$ where $k \geqq 1$ and no $x_{i}{ }^{e_{i}} x_{i+1}{ }^{e_{i+1}}=1$, some part of each factor remains after cancellation, whence $w \neq 1$. This proves that $X$ is a basis. (It is curious that Nielsen himself used at this point a different argument.) 
We conclude with two remarks. The first is a minor point, that Nielsen used a slightly different basis consisting of elements of the form

$$
x^{\prime}=\left(a_{1} \cdots a_{i-1} a_{i+1} \cdots a_{j-1}\right)\left(a_{i} a_{j} a_{i}^{-1} a_{j}^{-1}\right)\left(a_{1} \cdots a_{i-1} a_{i+1} \cdots a_{j-1}\right)^{-1} .
$$

It is easy to pass from one basis to the other by Nielsen transformations. The second point is that Nielsen treated only the case that the $G_{i}$ are all finite cyclic groups, and in this case gave a formula for the rank $r(N)=|X|$ of $N$. For the slightly more general case that all the groups $G_{i}$ are arbitrary finite groups, and hence that $\bar{G}$ is finite and $N$ again of finite rank, a formula for $r(N)$ can be recovered easily by counting how many $n$-tuples $a_{1}, \cdots, a_{n}$ yield non trivial elements $x \in X$. The result can be stated as a generalization of Schreier's index theorem. Assuming all the $G_{i}$ finite, define the 'free rank' of $G_{i}$ to be $r\left(G_{i}\right)=1-1 /\left|G_{i}\right|$ and $r(G)$ $=r\left(G_{1}\right)+\cdots+r\left(G_{n}\right)$. Then one has

$$
[G: N]=\frac{r(N)-1}{r(G)-1} .
$$

In Nielsen's case this is indeed a case of a classical formula for surface groups, for which a combinatorial proof is given in [2]. One may conjecture that such a formula holds for a subgroup $N$, not neccesarily normal, in a free product $G$ of some more extensive class of groups $G_{i}$ for which a reasonable definition of $r\left(G_{i}\right)$ can be provided.

Postscript (August, 1972). Mr. I. Chiswell has established that for $G$ and the function $r$ as defined above, the formula stated above holds for any subgroup $N$ of finite index in $G$, without the assumption that $N$ is normal.

\section{References}

[1] H. S. M. Coxeter and W. O. J. Moser, Generators and Relations for Discrete Groups, 2nd ed., Ergebisse der Mathematik und ihrer Grenzegbiete, Band 14. (Springer-Verlag, Berlin, Göttingen, Heidleberg, New York, 1965).

[2] A. Howard M. Hoare, Abraham Karrass and Donald Solitar, 'Subgroups of finite index of Fuchsian groups', Math. Z. 120 (1971), 289-298.

[3] A. M. Macbeath, Fuchsian Groups, (Lectures at Queen's College, Dundee, 1961.)

[4] Jakob Nilsen, 'The commutator group of the free product of cyclic groups', (Danish), Mat. Tidskr. B 1948 (1948), 49-56.

[5] Jakob Nielsen, 'A basis for subgroups of free groups', Math. Scand. 3 (1955), 31-43.

[6] R. A. Rankin, The Modular Group and its Subgroups. (Ramanujan Institute, 1969),

Université de Montpellier

Montpellier, France.

Present address:

University of Michigan

Ann Arbor, Michigan, USA 